\title{
Knowledge, attitudes and practices regarding human papillomavirus vaccination among young women attending a tertiary institution in Singapore
}

\author{
Qing Yuan Zhuang ${ }^{1}$, MBBs, Mmed, Ru Xin Wong $^{2}$, FRCR, Wei Ming Darren $\underline{C h e n}^{3}$, MBBs, Xiao Xuan $\underline{\text { Guo }}{ }^{3}$, MBBS, MmED
}

INTRODUCTION This study aimed to describe the knowledge, attitudes and practices of young women regarding human papillomavirus (HPV) vaccination.

METHODS We conducted a descriptive, cross-sectional, questionnaire-based study among female students at a tertiary institute in Singapore.

RESULTS A total of 255 questionnaires were completed and formed the basis of the analysis. 244 (95.7\%) of the total participants were of the age group 15-22 years. 252 (98.8\%) participants were unmarried and 240 (94.1\%) had never had sexual intercourse. Only 25 (9.8\%) women had received vaccination. Among the unvaccinated participants, 96 (41.7\%) had no intention to receive HPV vaccination and 62 of them cited lack of information as a major barrier to HPV vaccination. Knowledge of cervical cancer and HPV vaccination was also assessed and graded via a point system, with a maximum score of 14. Knowledge was found to be low, with a median score of 7 . There was a significant association between HPV vaccination uptake and the source from which they first heard about the vaccine $(p=0.007)$. Vaccinated respondents tended to first hear about it from their relatives and friends, as compared to unvaccinated respondents (60.0\% vs. $27.0 \%)$ CONCLUSION There is poor uptake of HPV vaccination amongst Singapore's susceptible youth as well as poor knowledge of cervical cancer and HPV vaccination. Public health education regarding cervical cancer and HPV vaccination is still needed and has to be targeted at not only respondents, but also their family and friends.

Keywords: cervical cancer, primary care, vaccination

\section{INTRODUCTION}

Cervical cancer is the fourth most common cancer among women worldwide, ${ }^{(1)}$ affecting 528,000 women annually and resulting in more than 270,000 deaths. ${ }^{(2)}$ It is currently the ninth most common cancer among Singaporean women, though the incidence rate has significantly declined over the last 40 years. ${ }^{(3)}$ The age-standardised incidence rate fell from 17.6 per 100,000 in 1972-1976 to 7.0 per 100,000 in 2007-2011. The age-standardised five-year observed survival rate for cervical cancer has also increased from $42.7 \%$ in $1973-1977$ to $63.6 \%$ in $2003-2007$. Cervical cancer has the eighth-highest cancer mortality rate in Singapore, with an agestandardised mortality rate of 2.5 per 100,000 in 2007-2011. ${ }^{(3)}$

Oncogenic human papillomavirus (HPV) infection is the necessary cause of cervical cancer. Worldwide, $70 \%$ of invasive cervical cancer cases are caused by HPV 16 or 18 , with HPV 16 being the most common type, detected in $55 \%$ of cases. This is followed by HPV 18 in $15 \%$ of cases. ${ }^{(4,5)}$ Additional related oncogenic HPV types make up an additional $18 \%$ of all cases.

HPV-related diseases incur substantial economic burdens in many countries. Among the HPV-related diseases, cervical cancer and cervical dysplasia are estimated to account for direct medical costs of USD 4.6 billion annually in the United States (US). ${ }^{(6)}$ In Singapore, the estimated total direct cost of cervical cancer and dysplasia was SGD 77.5 million over 25 years. ${ }^{(7)}$ Two HPV vaccines are currently licensed by the US Food and Drug Administration. The bivalent HPV vaccine (Cervarix $®)$ prevents
HPV 16 and 18, while the quadrivalent HPV vaccine (Gardasil $(\mathbb{)})$ prevents HPV 6, 11, 16 and 18. Prophylactic HPV vaccines have been shown to be safe, well-tolerated and highly efficacious in preventing persistent infections and cervical diseases associated with vaccine HPV types among young women. ${ }^{(8)}$ In Singapore, they have also been shown to be cost-effective and a good strategy to reduce the impact of HPV infection. ${ }^{(9)}$

The World Health Organization recommends that HPV vaccination should be introduced into national immunisation programmes in countries where prevention of cervical cancer is a public health priority and it is programmatically feasible, economically sustainable and cost-effective to do so. ${ }^{(10)}$ Although Singapore has yet to implement HPV vaccination in our national immunisation programme, there has been a publicity drive to increase awareness about HPV vaccination and the Health Promotion Board, Singapore, has published clear recommendations online. ${ }^{(11)}$ To the best of our knowledge, no published data regarding HPV vaccination uptake in Singapore is currently available.

This study aimed to survey the knowledge, attitudes and practices regarding HPV vaccination among young women in Singapore. Such data would be useful for the development of appropriate strategies for HPV vaccinations, and thus help reduce the morbidity and mortality associated with the disease. The study also aimed to sample the vaccination uptake rate in an appropriate target population and explore the factors that influence vaccination uptake rates.

${ }^{1}$ Division of Palliative Medicine, ${ }^{2}$ Division of Radiation Oncology, National Cancer Centre Singapore, ${ }^{3}$ SingHealth Polyclinics, Singapore

Correspondence: Dr Zhuang Qing Yuan, Registrar, Division of Palliative Medicine, National Cancer Centre Singapore, 11 Hospital Drive, Singapore 169610. zhuang.qingyuan@nccs.com.sg 


\section{METHODS}

This was a descriptive cross-sectional survey that specifically targeted students attending a tertiary institution in Singapore. The questionnaire was devised in consultation with family physician consultants and took into consideration educational materials from the Health Promotion Board that are readily available to the public (Appendix). We pretested the questionnaire on a small group for face validity. Institutional Review Board approval was obtained before the commencement of the study. We obtained a waiver of consent for children aged $<21$ years, as our target population was aged 15-26 years.

As this was an initial exploratory study, we utilised convenience non-random sampling to recruit participants. Surveys were anonymised and verbal, informed consent was obtained before commencement. The survey was self-administered to decrease the risk of self-censorship by participants, but researchers were available to assist participants and clarify any doubts. After completing the questionnaire, participants were given pamphlets on cervical cancer and HPV vaccination to create awareness. Information collected included sociodemographic data and HPV vaccination status; the 14 survey questions objectively quantified participants' knowledge of facts on cervical cancer, risk factors, vaccination eligibility and the need for subsequent follow-up. The questionnaire was scored based on participants' knowledge of cervical cancer and HPV vaccination; it also evaluated participants according to their perceived barriers to vaccination. A correct answer was allocated one point whereas wrong answers were not allocated points; participants could achieve a maximum score of 14 points.

PASW Statistics version 18.0 (SPSS Inc, Chicago, IL, USA) was used for all data entry and analyses. A p-value $<0.05$ was considered statistically significant. All descriptive statistics were presented as proportions and compared using chi-square test. The median knowledge score was analysed with Mann-Whitney $U$ test and Kruskal-Wallis test.

\section{RESULTS}

Out of the 266 questionnaires that were distributed, 255 completed questionnaires were included in this study, while 11 were excluded due to incomplete data. The majority of the respondents $(62.0 \%)$ were young women aged $15-18$ years, while the second-largest group of respondents (33.7\%) was aged 19-22 years. The ethnicity distribution of the respondents closely resembled the national demographic distribution. Almost all the respondents were unmarried $(98.8 \%$ ) and a majority indicated that they had never had sexual intercourse (94.1\%). The demographic characteristics of the study sample are shown in Table I.

Participants' knowledge of cervical cancer and HPV vaccination was assessed and scored for quantitative assessment. The mean score was 7.09 (95\% confidence interval [CI] 4.12-10.06). The median score was 7; the lowest score was 4 and the highest was 13 (Fig. 1). Median knowledge scores crosstabulated against history of sexual intercourse, marital status, age group and HPV vaccination status showed no statistically significant difference (Table II).

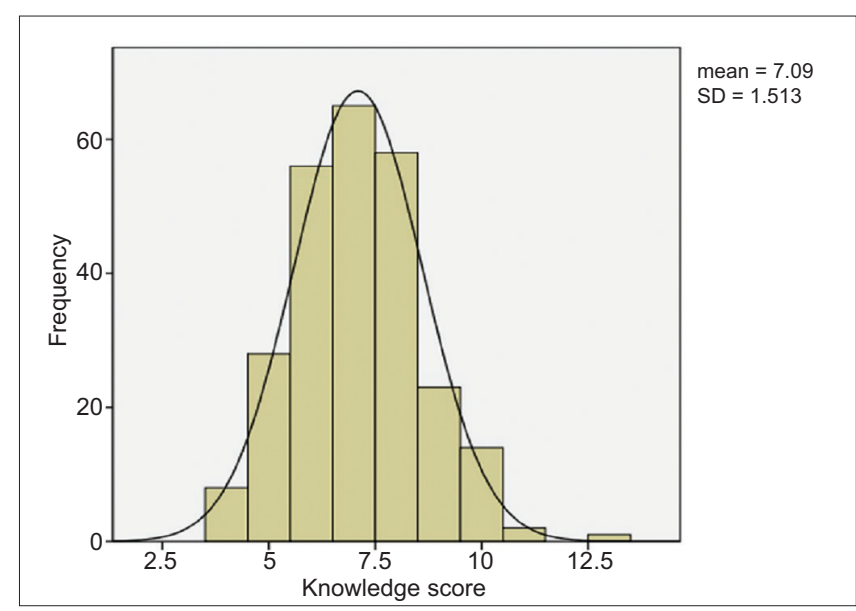

Fig. 1 Histogram shows the distribution of respondents' knowledge scores.

Table I. Participant demographics and source of information requested in the survey $(n=255)$.

\begin{tabular}{|c|c|}
\hline Characteristic & No. (\%) \\
\hline \multicolumn{2}{|l|}{ Age group (yr) } \\
\hline $15-18$ & $158(62.0)$ \\
\hline $19-22$ & $86(33.7)$ \\
\hline $23-26$ & $7(2.7)$ \\
\hline $27-30$ & $3(1.2)$ \\
\hline $31-34$ & $1(0.4)$ \\
\hline \multicolumn{2}{|l|}{ Ethnicity } \\
\hline Chinese & $194(76.1)$ \\
\hline Malay & $36(14.1)$ \\
\hline Indian & $15(5.9)$ \\
\hline Others & $10(3.9)$ \\
\hline \multicolumn{2}{|l|}{ Religion } \\
\hline Buddhist & $66(25.9)$ \\
\hline Christian/Catholic & $60(23.5)$ \\
\hline Muslim & $45(17.6)$ \\
\hline Taoist & $11(4.3)$ \\
\hline Hindu & $7(2.7)$ \\
\hline Others & $1(0.4)$ \\
\hline None & $65(25.5)$ \\
\hline \multicolumn{2}{|l|}{ Marital status } \\
\hline Single & $252(98.8)$ \\
\hline Married & $3(1.2)$ \\
\hline \multicolumn{2}{|c|}{ History of sexual intercourse } \\
\hline Yes & $15(5.9)$ \\
\hline No & $240(94.1)$ \\
\hline \multicolumn{2}{|l|}{ Source of information } \\
\hline Mass media & $89(34.9)$ \\
\hline Healthcare workers & $18(7.1)$ \\
\hline Relatives and friends & $77(30.2)$ \\
\hline Others & $71(27.8)$ \\
\hline
\end{tabular}

Attitudes and practices toward and perceived barriers to HPV vaccination were also evaluated. Notably, only $9.8 \%$ of the respondents had been vaccinated. The majority $(88.4 \%)$ of the 230 unvaccinated individuals perceived HPV vaccination to be effective. However, only 134 (58.3\%) of them had the intention to receive HPV vaccination. Of the 96 (41.7\%) individuals who 


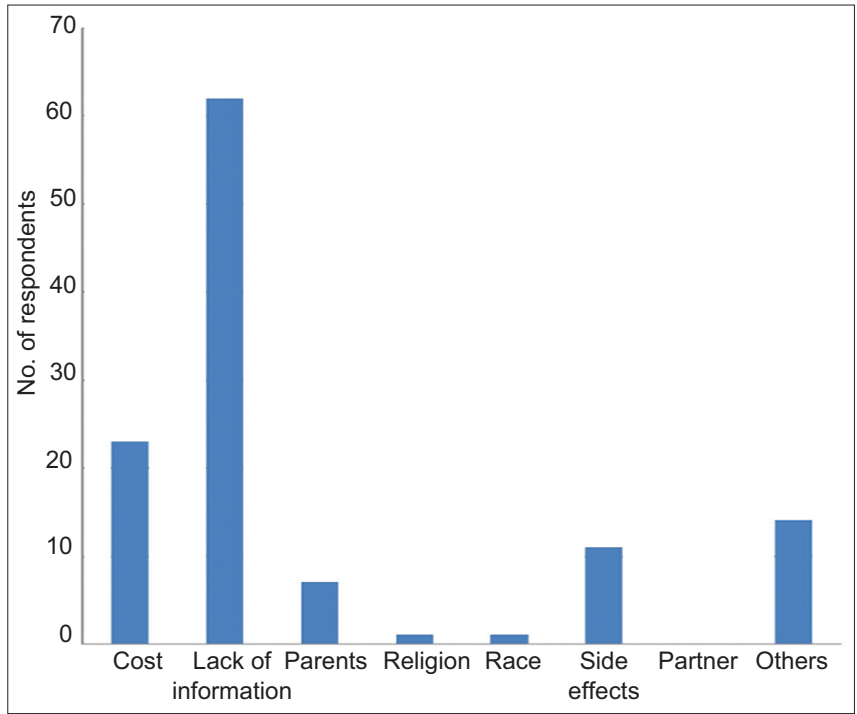

Fig. 2 Graph shows barriers to vaccination in respondents who did not intend to receive human papillomavirus vaccination. More than one option could be chosen.

had no intention to receive HPV vaccination, 62 cited lack of information as a major barrier (Fig. 2).

We analysed the vaccination status of respondents against demographic characteristics of the study sample and found a statistical difference in the source of respondents' information about HPV vaccination $(p=0.007)$. Respondents who had been vaccinated tended to first hear about HPV vaccination from relatives and friends, as compared to unvaccinated respondents $(60.0 \%$ vs. $27.0 \%$ ). Of those who were unvaccinated, $29.6 \%$ chose the option 'others' and the majority indicated that they first heard about HPV vaccination during the survey. We also noted that a large proportion of the unvaccinated respondents were aged 15-18 years (Table III).

\section{DISCUSSION}

Most respondents in the present study were aged 15-22 years, which is the target population for HPV vaccination. Furthermore, these respondents were largely unmarried and reported never having had sexual intercourse (i.e. no exposure to HPV), and hence were prime respondents for the introduction of HPV vaccination. Among the study population, uptake of vaccination was poor at $9.8 \%$. To the best of our knowledge, no other studies have been conducted in Singapore to evaluate uptake of vaccination within the target population. In addition, up to $42.5 \%$ of unvaccinated respondents declined vaccination.

Improving the uptake of vaccination is of utmost importance, as vaccination is cost-effective compared to the significant economic burden of cervical cancer. Various studies have shown the potential impact of HPV vaccination on cervical cancer and its precursors; vaccination of an entire cohort of females before their sexual debut could significantly reduce the lifetime risk of cervical cancer. ${ }^{(12,13)}$ A recent systematic review regarding barriers to and facilitators of HPV vaccination in young women in high-income countries showed that HPV vaccination uptake was determined by policymakers, healthcare professionals and parents. ${ }^{(14)}$ Findings also suggested that young women are predominantly passive recipients
Table II. Comparison of knowledge scores and patient demographics.

\begin{tabular}{|c|c|c|c|}
\hline Parameter & No. & $\begin{array}{c}\text { Median } \\
\text { knowledge } \\
\text { score }\end{array}$ & p-value \\
\hline History of sexual intercourse & & & 0.236 \\
\hline Yes & 15 & 8 & \\
\hline No & 240 & 7 & \\
\hline Age group (yr) & & & 0.055 \\
\hline $15-18$ & 158 & 7 & \\
\hline $19-22$ & 86 & 7 & \\
\hline $23-26$ & 7 & 6 & \\
\hline $27-30$ & 3 & 8 & \\
\hline $31-34$ & 1 & 10 & \\
\hline Marital status & & & NA \\
\hline Single & 252 & 7 & \\
\hline Married & 3 & 8 & \\
\hline HPV vaccination status & & & 0.532 \\
\hline Yes & 25 & 8 & \\
\hline No & 230 & 7 & \\
\hline
\end{tabular}

HPV: human papillomavirus; NA: not applicable

Table III. Comparison of vaccination status and respondent characteristics.

\begin{tabular}{|c|c|c|c|}
\hline \multirow[t]{2}{*}{ Parameter } & \multicolumn{2}{|c|}{ No. (\%) } & \multirow[t]{2}{*}{ p-value } \\
\hline & $\begin{array}{l}\text { Vaccinated } \\
(n=25)\end{array}$ & $\begin{array}{l}\text { Not vaccinated } \\
(n=230)\end{array}$ & \\
\hline Source of information & & & 0.007 \\
\hline Mass media & $5(20.0)$ & $84(36.5)$ & \\
\hline Healthcare worker & $2(8.0)$ & $16(7.0)$ & \\
\hline Relatives and friends & $15(60.0)$ & $62(27.0)$ & \\
\hline Others & $3(12.0)$ & $68(29.6)$ & \\
\hline Age group (yr) & & & 0.005 \\
\hline $15-18$ & $10(40.0)$ & $148(64.3)$ & \\
\hline $19-22$ & $12(48.0)$ & $74(32.2)$ & \\
\hline $23-26$ & $1(4.0)$ & $6(2.6)$ & \\
\hline $27-30$ & $2(8.0)$ & $1(0.4)$ & \\
\hline $31-34$ & 0 & $1(0.4)$ & \\
\hline Ethnicity & & & 0.438 \\
\hline Chinese & $21(84.0)$ & $173(75.2)$ & \\
\hline Malay & $2(8.0)$ & $34(14.8)$ & \\
\hline Indian & $1(4.0)$ & $14(6.1)$ & \\
\hline Others & $1(4.0)$ & $9(3.9)$ & \\
\hline Religion & & & 0.695 \\
\hline Buddhist & $8(32.0)$ & $58(25.2)$ & \\
\hline Christian/Catholic & $7(28.0)$ & $53(23.0)$ & \\
\hline Muslim & $2(8.0)$ & $43(18.7)$ & \\
\hline Taoist & $2(8.0)$ & $9(3.9)$ & \\
\hline Hindu & 0 & $7(3.0)$ & \\
\hline Others & 0 & $1(0.4)$ & \\
\hline None & $6(24.0)$ & $59(25.7)$ & \\
\hline $\begin{array}{l}\text { History of sexual } \\
\text { intercourse }\end{array}$ & & & 0.171 \\
\hline Yes & $3(12.0)$ & $12(5.2)$ & \\
\hline No & $22(88.0)$ & $218(94.8)$ & \\
\hline
\end{tabular}


of the HPV vaccine. Parents appear to retain their role in decisionmaking and healthcare professionals appear to reinforce this role. ${ }^{(15)}$

Singapore has a comprehensive National Childhood Immunisation Programme (NCIP) with a good track record, which has been shown to have excellent immunisation coverage in infants, preschool and school children. ${ }^{(16)}$ Further studies on the feasibility and cost-effectiveness of HPV vaccination could be conducted on our local population to consider its inclusion in the NCIP as a first step to improving its uptake. Physicians also need to play a part in recommending vaccination, and more studies should be done to review interventions that increase such practices.

The present study showed that knowledge regarding cervical cancer and HPV vaccination was generally low, with a median score of 7 out of a possible 14. Notably, respondents' level of knowledge was low regarding the prevalence of cervical cancer and various important risk factors for cervical cancer such as smoking, early age of first sexual intercourse and history of sexually transmitted disease. This is of concern, especially as our study identified lack of knowledge as the major barrier for respondents declining HPV vaccination. Respondents who had received HPV vaccination were also found to have higher mean and median knowledge scores (7.24 vs. 7.09 and 8 vs. 7 respectively), although these were not found to be statistically significant. Similarly, a recent systematic review examining the association between HPV vaccination and HPV knowledge in the United Kingdom found that knowledge surrounding HPV was generally poor and vaccinated respondents (or those with the intent to receive vaccination) had higher levels of knowledge than unvaccinated respondents. ${ }^{(17)}$ Educational interventions have been shown to improve knowledge about HPV and cervical cancer. ${ }^{(18)}$ However, strong evidence is lacking that any specific educational intervention can influence HPV vaccine uptake, and further studies are required..$^{(19)}$

We found that the subset of respondents who received HPV vaccination tended to first hear about it from relatives and friends $(p=0.007)$. Similar studies have suggested that social sources of information are important for increasing perceived vaccine effectiveness and requests for the vaccine. ${ }^{(20,21)}$ Hence, we hypothesised that relatives and friends provide the necessary push factor to encourage the uptake of vaccination. Therefore, campaigns designed to improve awareness of HPV vaccination should not just focus on the target audience, but also include their family and friends. To the best of our knowledge, this study is the first to evaluate HPV vaccination practices and uptake among Singaporean women. However, several limitations were present. As it was a cross-sectional study, we were unable to establish any causal relationships between the knowledge or attitude of the respondents and their practices. The convenience sampling method of administering the questionnaire may also introduce selection bias, which can affect the generalisability of the study. Additionally, we only surveyed students from one tertiary institute, but attitudes and barriers may differ between institutes and age groups, affecting the generalisability of this study to the female population in Singapore. The small sample size also limited our statistical analyses. With further governmental interventions regarding HPV vaccination, further studies will be needed to reflect any changes in knowledge, attitudes, perceived barriers and practices.
In conclusion, HPV vaccination has poor uptake amongst Singapore's susceptible youth, and knowledge regarding HPV and HPV vaccination is lacking. Public health education on cervical cancer and HPV vaccination is still needed, and has to be targeted not just at the respondents, but also their family and friends.

\section{ACKNOWLEDGEMENT}

This study was funded by the SingHealth Family Medicine Residency Programme.

\section{REFERENCES}

1. International Agency for Research on Cancer. Latest world cancer statistics [online] 2013. Available at: https://www.iarc.fr/en/media-centre/pr/2013/ pdfs/pr223_E.pdf. Accessed November 14, 2015.

2. World Health Organization. Human papillomavirus (HPV) and cervical cancer [online]. Available at: http://www.who.int/mediacentre/factsheets/ fs380/en/. Accessed November 14, 2015.

3. National Registry of Diseases Office, Singapore. Health Factsheet: Trends of Cervical Cancer in Singapore 2007-2011. Available at: https://www. nrdo.gov.sg/docs/librariesprovider3/Publications-Cancer/health_factsheet_ cervical_2013apr.pdf?sfvrsn=0. Accessed November 27, 2015.

4. Walboomers JM, Jacobs MV, Manos MM, et al. Human papillomavirus is a necessary cause of invasive cervical cancer worldwide. J Pathol 1999; 189:12-9.

5. Smith JS, Lindsay L, Hoots B, et al. Human papillomavirus type distribution in invasive cervical cancer and high-grade cervical lesions: a meta-analysis update. Int J Cancer 2007; 121:621-32.

6. Lipsy RJ. Assessing the short-term and long-term burden of illness in cervical cancer. Am J Manag Care 2008; $14(6$ Suppl 1):S177-84.

7. Low JJ, Ko Y, Ilancheran A, et al. Health and economic burden of HPVrelated diseases in Singapore. Asian Pac J Cancer Prev 2012; 13:305-8.

8. Lee VJ, Tay SK, Teoh YL, Tok MY. Cost-effectiveness of different human papillomavirus vaccines in Singapore. BMC Public Health 2011; 11:203.

9. Lu B, Kumar A, Castellsagué X, Giuliano AR. Efficacy and safety of prophylactic vaccines against cervical HPV infection and diseases among women: a systematic review \& meta-analysis. BMC Infectious Dis 2011; 11:13.

10. World Health Organization WHO position on HPV vaccines. Vaccine 2009; 27:7236-7.

11. Health Promotion Board, Singapore. FAQs on HPV and HPV immunisation. Available at: http://www.hpb.gov.sg/HOPPortal/health-article/8768. Accessed November 20, 2015

12. Elbasha EH, Dasbach EJ, Insinga RP. Model for assessing human papillomavirus vaccination strategies. Emerg Infect Dis 2007; 13:28-41.

13. Barnabas RV, Laukkanen P, Koskela P, et al. Epidemiology of HPV 16 and cervical cancer in Finland and the potential impact of vaccination: mathematical modelling analyses. PLoS Med 2006; 3:e138.

14. Ferrer HB, Trotter C, Hickman M, Audrey S. Barriers and facilitators to HPV vaccination of young women in high-income countries: a qualitative systematic review and evidence synthesis. BMC Public Health 2014; 14:700.

15. Rambout L, Tashkandi M, Hopkins L, Tricco AC. Self-reported barriers and facilitators to preventive human papillomavirus vaccination among adolescent girls and young women: a systematic review. Prev Med 2014; 58:22-32.

16. Liew F, Ang LW, Cutter J, James L, Goh KT. Evaluation on the effectiveness of the national childhood immunisation programme in Singapore, 19822007. Ann Acad Med Singapore 2010; 39:532-10.

17. Coles VA, Patel AS, Allen FL, Keeping ST, Carroll SM. The association of human papillomavirus vaccination with sexual behaviours and human papillomavirus knowledge: a systematic review. Int J STD AIDS 2015; 26:777-88.

18. Sossauer G, Zbinden M, Tebeu PM, et al. Impact of an educational intervention on women's knowledge and acceptability of human papillomavirus self-sampling: a randomized controlled trial in Cameroon. PloS One 2014; 9:e109788.

19. Fu LY, Bonhomme LA, Cooper SC, Joseph JG, Zimet GD. Educational interventions to increase HPV vaccination acceptance: a systematic review. Vaccine 2014; 32:1901-20.

20. Casillas A, Singhal R, Tsui J, et al. The impact of social communication on perceived HPV vaccine effectiveness in a low-income, minority population. Ethn Dis $2011 ; 21: 495-501$

21. Javanbakht M, Stahlman S, Walker S, et al. Provider perceptions of barriers and facilitators of HPV vaccination in a high-risk community. Vaccine 2012; 30:4511-6. 


\section{APPENDIX}

\section{Questionnaire}

Circle as appropriate.

\section{Demographic information}

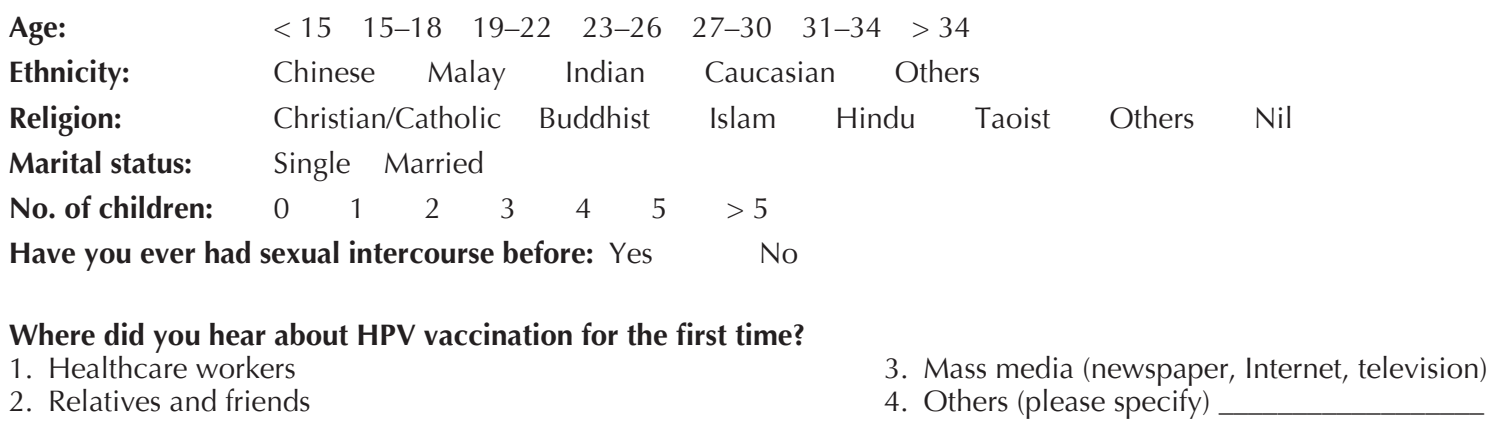

\section{About cervical cancer}

Among the top 10 cancers in women in Singapore, cervical cancer ranks at number
(a) $1-3$
(b) 4-6
(c) $7-10$

What are risk factors for cervical cancer? (Multiple answers allowed.)
1. Family history of cervical cancer
6. Sexual transmitted disease
2. Multiple childbirth
7. Smoking
3. Multiple sexual partners
8. Early age of first sexual intercourse $(<18$ years old $)$
4. Vaginal yeast infection
9. Diet
5. Certain strains of genital human papillomavirus infection
10. Not using a condom

\section{About HPV vaccination}

HPV vaccination is available at polyclinics. True False

Where would you prefer to receive the HPV vaccination?
1. Well women clinic in polyclinic
3. Private clinic
2. Gynaecological clinic/hospital
4. No preference

How much does the HPV vaccine cost in Singapore (per dose)?

$\$ 0 \quad \$ 1-50 \quad \$ 51-100 \quad \$ 101-200 \quad \$ 201-300 \quad>\$ 300$

Is the cost of HPV vaccination claimable from Medisave? Yes No

HPV vaccination is only for women at high risk of cervical cancer. True False

HPV vaccine is only for women $<\mathbf{2 6}$ years old. True False

Women who have received HPV vaccination no longer need to go for regular Pap smears. True False

Have you had HPV vaccination before? Yes No

If ' $\mathrm{No}^{\prime}$, are you considering going for the vaccination? Yes

No

Vaccination is effective in preventing cervical cancer. True False

If ' $\mathrm{No}^{\prime}$, what the reasons for not taking the vaccination? (Multiple answers allowed.)
1. Side effects are too high
5. My parents do not allow me to take it
2. My ethnicity does not permit HPV vaccination
6. I do not have enough information about HPV vaccine
3. My religion does not permit HPV vaccination
7. Too costly
4. My partner does not allow me to take it
8. Other reasons: 